\title{
Factores limitantes en la negociación internacional de productos farmacéuticos de las PYMES: importadora JORUA CIA LTDA
}

\section{Limiting factors in the international negotiation of pharmaceutical products of PYMES: importer JORUA CIA LTDA}

\author{
Dayse Cevallos Villegas ${ }^{1}$ \\ dayse cevallos26@hotmail.com \\ https://orcid.org/0000-0002-6206-3598 \\ Fernando Ronquillo Murrieta ${ }^{2}$ \\ f ronquillom@hotmail.com \\ https://orcid.org/0000-0001-7593-5028
}

Recibido: 28/9/2021, Aceptado: 28/12/2021

\begin{abstract}
RESUMEN
El propósito del trabajo es determinar las limitaciones internacionales que predominan en el proceso de la importación de productos farmacéuticos en las pymes (Importadora Jorua Cia. Ltda.). Se utiliza el método analítico - sintético y como técnicas de recolección de datos, la investigación documental y la encuesta. Los resultados con base en la encuesta efectuada al personal administrativo, contable en su totalidad, con un $65 \%$ conocen sobre los factores limitantes en la negociación internacional, y un $91 \%$ de los trabajadores menciona la dificultad que lleva el proceso de importar para la empresa. Como conclusión se encuentran diferentes factores limitantes en la negociación internacional principalmente en la importación de productos, y se manifiestan varias situaciones en lo político, económico, social y tecnológico demostrando una estabilidad interesante que aportan al desarrollo y crecimiento del sector.
\end{abstract}

Palabras clave: Comercio Internacional, Empresa, Gobierno, Importación, Mercado

\section{ABSTRACT}

The purpose of the work is to determine the international limitations that predominate in the importation process of pharmaceutical products in SMEs (Importadora Jorua Cia. Ltda.). The analytical - synthetic method is used and as data collection techniques, documentary research and the survey The results based on the survey carried out to the administrative staff, accounting in its

\footnotetext{
1 Magíster en Administración de Empresas con mención en Negocios Internacionales, Universidad Tecnológica Empresarial de Guayaquil, Ecuador

${ }^{2}$ Magíster en Negocios Internacionales, Universidad Tecnológica Empresarial de Guayaquil, Ecuador
} 


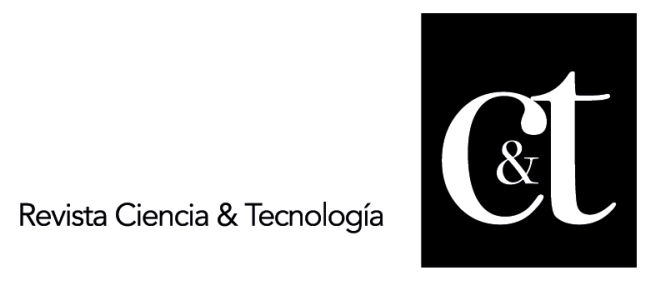

No. 33, 31 de enero de 2022

ISSN impreso: 1390 - 6321

ISSN online: 2661 - 6734

entirety, with $65 \%$ known about the limiting factors in the negotiation international, and $91 \%$ of the workers mentioned the difficulty of the importing process for the company. As a conclusion, there are different limiting factors in international negotiation, mainly in the importation of products, and various political, economic, social and technological situations are manifested, demonstrating an interesting stability that they contribute to the development and growth of the sector.

Keywords: International Trade, Business, Government, Import, Market.

\section{Introducción}

El presente trabajo hace una exposición de estudio a los factores limitantes en la negociación internacional de las pymes, cuya situación es compleja ya que en la actualidad existen diferentes limitaciones como por ejemplo la pandemia del virus covid-19; pero una de las barreras de mayor peso son las del Estado ya que constantemente se manifiestan nuevas restricciones que imposibilitan a las empresas a ejecutar las importaciones correspondientes; conllevando por lo consiguiente a obtener pérdidas financieras, faltante de productos y el desarrollo internacional de las empresas.

Otra restricción importante tratada en el trabajo se refiere al tipo de empresa estudiada: nos limitamos a empresas de pequeño tamaño. En efecto, el potencial de importar de la pequeña y mediana empresa Importadora Jorua Cía. Ltda., es un asunto de creciente de interés para el análisis económico local. Tan sólo un pequeño porcentaje de las pequeñas y medianas empresas realizan actividades internacionales y, sin embargo, su contribución a la importación total es ya muy alta en diferentes países.

En un mundo cada vez más integrado en lo económico y comercial, la competitividad internacional ha dejado de relacionarse netamente con el factor precio para abarcar cuestiones más amplias como la innovación, diseño, calidad, la capacidad de adaptación y la cercanía a los consumidores.

La idea de una empresa competitiva se asocia con su capacidad de operar internacionalmente en la importación de bienes, de tal modo que la internacionalización de la empresa se asume como un requisito imprescindible para el crecimiento y la rentabilidad.

El alcance del trabajo es determinar, fundamentar, diagnosticar y proponer estrategias que ayuden a facilitar la negociación internacional de los fármacos en las pymes (Importadora Jorua Cía. Ltda.), y obtener mayor crecimiento en el mercado de la importación.

Ante todo, se debe conocer el concepto de una investigación el cual es el procedimiento de indagación y curiosidad que se evidencia mediante la evidencia física que se plasma en un informe con la ayuda de la información recolectada por el cual se llega a obtener conocimiento científico, aunque no existe un método $100 \%$ infalible que pueda eliminar el margen de error que pueda darse en la elaboración del escrito. (Stenhouse, 2018)

En un sentido global, el autor Tomás Gallego Izquierdo (2007) los métodos son un conjunto de procedimientos que se llevan a cabo con la finalidad de encontrar la verdad y mostrarla, su principal objetivo es la solución de problemas mediante el uso y aplicación de técnicas e instrumentos. 


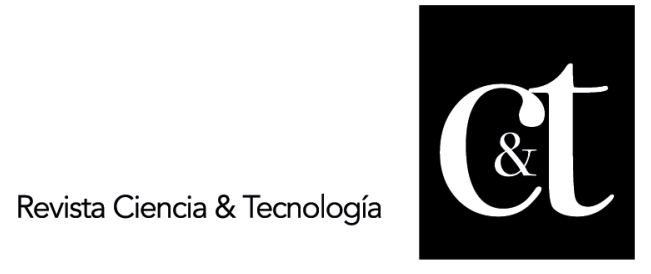

No. 33, 31 de enero de 2022

ISSN impreso: 1390 - 6321

ISSN online: 2661 - 6734

Para la presente investigación, se considera como objeto de estudio a la Importadora Jorua Cía. Ltda. Constituida en la ciudad de Babahoyo, provincia de Los Ríos y la población está definida por los empleados, jefes y gerente. En relación a la muestra, se considera el personal de la Importadora JORUA Cía. Ltda., seleccionando un total de 123 empleados, 7 jefes y 3 gerentes.

La Importadora Jorua Cía. Ltda. dedicada a la venta al por menor de papelería como lápices, bolígrafos, papel, etc. Esta tuvo su inicio de actividad un 23 de octubre de 2000, con un domicilio tributario en Guayaquil y una sucursal en la ciudad de Babahoyo.

Además, entre otras de sus actividades económicas se encuentra la venta al por menor de productos farmacéuticos en el establecimiento correspondiente ubicada en la Provincia de Los Ríos, ciudad de Babahoyo.

De acuerdo a Valencia (2017) La compañía se encuentra compuesta por 4 departamentos:

$>$ Administración

$>$ Gerente

> Departamento de Almacén e Inventarios

> Departamento de Compras y Ventas

Específicamente a partir de marzo del 2020, algunos países introdujeron restricciones a la exportación de productos farmacéuticos debido al COVID-19. A inicio de mayo, un total de 70 países aplicaron las medidas de ese tipo.

En la tabla 1 se vislumbrará las medidas que se vieron adoptadas por los principales proveedores de América Latina y el Caribe, incluyendo otros países de la misma región. ((CEPAL), 2020)

Tabla 1 Países y agrupaciones seleccionados: restricciones a la exportación de productos médicos introducidas en respuesta al COVID-19, al 28 de abril de 2020.

\begin{tabular}{|c|c|c|c|}
\hline $\begin{array}{l}\text { País o } \\
\text { Agrupación }\end{array}$ & Tipo de medida & $\begin{array}{l}\text { Fecha } \\
\text { de } \\
\text { entrega } \\
\text { en } \\
\text { vigor }\end{array}$ & Duración \\
\hline \multicolumn{4}{|c|}{ Extra regionales } \\
\hline $\begin{array}{l}\text { Estados } \\
\text { Unidos }\end{array}$ & $\begin{array}{l}\text { Se prohíbe la } \\
\text { exportación de } \\
\text { respiradores N95 y } \\
\text { similares, } \\
\text { mascarillas y } \\
\text { guantes quirúrgicos } \\
\text { si la autorización de } \\
\text { la Agencia Federal } \\
\text { para el manejo de } \\
\text { Emergencias (FEMA) }\end{array}$ & $\begin{array}{l}10 \text { de } \\
\text { abril } \\
\text { del } \\
2020\end{array}$ & $\begin{array}{l}\text { Cuatro } \\
\text { meses }\end{array}$ \\
\hline
\end{tabular}


No. 33, 31 de enero de 2022

ISSN impreso: 1390 - 6321

ISSN online: 2661 - 6734

\begin{tabular}{|c|c|c|c|}
\hline $\begin{array}{l}\text { Unión } \\
\text { Europea }\end{array}$ & $\begin{array}{l}\text { Se exige una licencia } \\
\text { para la exportación } \\
\text { de mascarillas y } \\
\text { otros equipos de } \\
\text { protección, personal } \\
\text { fuera de la Unión } \\
\text { Europea, la } \\
\text { Asociación Europea } \\
\text { de Libre Comercio y } \\
\text { los Balcanes }\end{array}$ & $\begin{array}{l}14 \\
\text { marzo } \\
2020\end{array}$ & $\begin{array}{l}\text { Seis } \\
\text { semanas; } \\
\text { prorrogada } \\
\text { el } 26 \text { de } \\
\text { abril por } 30 \\
\text { días }\end{array}$ \\
\hline Suiza & $\begin{array}{l}\text { Se exige una } \\
\text { autorización para } \\
\text { exportar equipos de } \\
\text { protección personal, } \\
\text { excepto la Unión } \\
\text { Europea, el Reino } \\
\text { Unido y la Asociación } \\
\text { Europea de Libre } \\
\text { Comercio }\end{array}$ & $\begin{array}{l}26 \\
\text { marzo } \\
2020\end{array}$ & $\begin{array}{l}\text { No se } \\
\text { indica }\end{array}$ \\
\hline India & $\begin{array}{lr}\text { Se prohíbe } & \text { la } \\
\text { exportación } & \text { de } \\
\text { mascarillas, otros } \\
\text { equipos } & \text { de } \\
\text { protección personal, } \\
\text { ventiladores } \\
\text { mecánicos, kits de } \\
\text { diagnósticos } \\
\text { desinfectante }\end{array}$ & $\begin{array}{l}\text { Entre el } \\
25 \text { de } \\
\text { febrero } \\
\text { y el } 4 \\
\text { de abril } \\
\text { del } \\
2020\end{array}$ & Indefinida \\
\hline \multicolumn{4}{|c|}{ Regionales } \\
\hline Argentina & $\begin{array}{l}\text { Se exige un permiso } \\
\text { para la exportación } \\
\text { de ventiladores } \\
\text { mecánicos y sus } \\
\text { partes, y de diversos } \\
\text { desinfectantes, } \\
\text { equipos de } \\
\text { protección personal } \\
\text { y aparatos e } \\
\text { insumos médicos }\end{array}$ & $\begin{array}{l}19 \text { de } \\
\text { marzo } \\
\text { de } \\
2020 \text { y } \\
28 \text { de } \\
\text { marzo } \\
2020\end{array}$ & $\begin{array}{l}\text { Mientras } \\
\text { dure el } \\
\text { estado de } \\
\text { emergencia } \\
\text { pública } \\
\text { sanitaria }\end{array}$ \\
\hline Brasil & \begin{tabular}{l}
\multicolumn{3}{l}{ Se exige una licencia } \\
especial para la \\
exportación \\
mascarilla y otros \\
equipos de \\
protección personal, \\
ventiladores \\
mecánicos,
\end{tabular} & $\begin{array}{l}18 \\
\text { marzo } \\
\text { de } \\
2020\end{array}$ & $\begin{array}{l}\text { No se } \\
\text { indica }\end{array}$ \\
\hline
\end{tabular}

Cevallos y Ronquillo. Factores limitantes en la negociación internacional de productos farmacéuticos de las PYMES: importadora JORUA CIA LTDA 


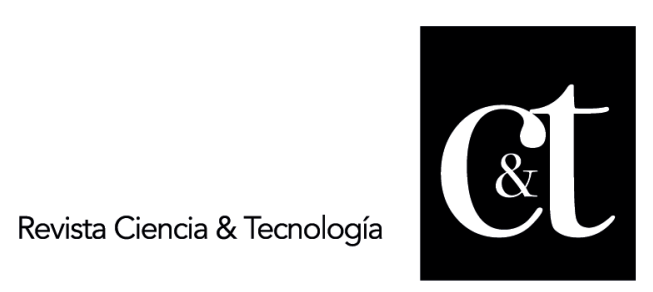

No. 33, 31 de enero de 2022

ISSN impreso: 1390 - 6321

ISSN online: 2661 - 6734

\begin{tabular}{|c|c|c|c|}
\hline & $\begin{array}{l}\text { desinfectantes y kits } \\
\text { de diagnósticos, } \\
\text { entre otros }\end{array}$ & & \\
\hline Colombia & $\begin{array}{l}\text { Se prohíbe la } \\
\text { exportación de } \\
\text { mascarillas y otros } \\
\text { equipos de } \\
\text { protección personal, } \\
\text { desinfectantes, } \\
\text { ciertos } \\
\text { medicamentos, } \\
\text { ventiladores } \\
\text { mecánicos y otros } \\
\text { equipos médicos }\end{array}$ & $\begin{array}{l}22 \\
\text { marzo } \\
\text { del } \\
2020\end{array}$ & Seis meses \\
\hline Costa Rica & $\begin{array}{lr}\text { Se establece } & \text { una } \\
\text { licencia } & \text { de } \\
\text { exportación } & \text { para } \\
\text { mascarillas y } & \text { otros } \\
\text { equipos } & \text { de } \\
\text { protección } & \text { de } \\
\text { personal. } & \end{array}$ & $\begin{array}{l}10 \text { abril } \\
\text { del } \\
2020\end{array}$ & $\begin{array}{l}\text { Mientras } \\
\text { dure el } \\
\text { estado de } \\
\text { emergencia } \\
\text { nacional }\end{array}$ \\
\hline Paraguay & $\begin{array}{l}\text { Se exigen licencias } \\
\text { de exportación de } \\
\text { alcohol en gel y } \\
\text { mascarillas } \\
\text { quirúrgicas }\end{array}$ & $\begin{array}{l}13 \text { de } \\
\text { marzo } \\
2020\end{array}$ & Un año \\
\hline Perú & $\begin{array}{l}\text { Se requiere la } \\
\text { opinión favorable del } \\
\text { Ministerio de Salud } \\
\text { para la exportación } \\
\text { de mascarillas y } \\
\text { otros equipos de } \\
\text { protección personal. }\end{array}$ & $\begin{array}{l}8 \text { abril } \\
\text { del } \\
2020\end{array}$ & $\begin{array}{l}\text { Mientras } \\
\text { dure el } \\
\text { estado de } \\
\text { emergencia } \\
\text { sanitaria }\end{array}$ \\
\hline
\end{tabular}

Nota. La información de la tabla representa las medidas adoptadas para la exportación de productos por los países Extra regionales y regionales debido a la emergencia sanitaria (covid-19).

Fuente: CEPAL, 2020

\section{Análisis Nacional}

El segmento del mercado farmacéutico del país ecuatoriano está dividido en el sector privado y el sector público; en el privado los medicamentos se comercializan en las farmacias, también en consultorios particulares así como en las grandes cadenas farmacéuticas y en el público se abastece mediante las compras públicas de fármacos. 


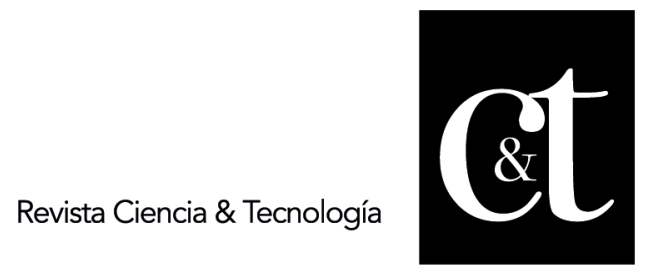

No. 33, 31 de enero de 2022

ISSN impreso: 1390 - 6321

ISSN online: 2661 - 6734

Las grandes cadenas farmacéuticas establecidas en el país cuentas con una gran variedad de medicamentos para todo tipo de malestar, es decir, ofrecen medicamentos de uso básico, uso continuo además ofrecen otros artículos de ventas para la higiene personal, esto ofrece a las comercializadoras equilibrar el crecimiento de ventas obtenidos. (Bank watch ratings S.A , 2019)

En su gran mayoría, el sector farmacéutico es importador, debido a que la materia prima que se utiliza en la fabricación de algunos medicamentos locales se debe de importar, así como otros medicamentos que se producen fuera.

En años anteriores (2015, 2016 y 2017) el sector Químico - farmacéutico hace hincapié en que dicho sector farmacéutico es altamente negativo, por ello se debe plantear la accesibilidad de los medicamentos que se elaboren en el territorio ecuatoriano. En el sector privado realizaron peticiones a los entes reguladores con la finalidad de garantizar la calidad de los productos además de la extinción de ciertos aranceles para la importación de materias primas importadas y estabilidad normativa y jurídica. (González, 2017)

Ilustración 1 Importaciones y Exportaciones de Medicamentos

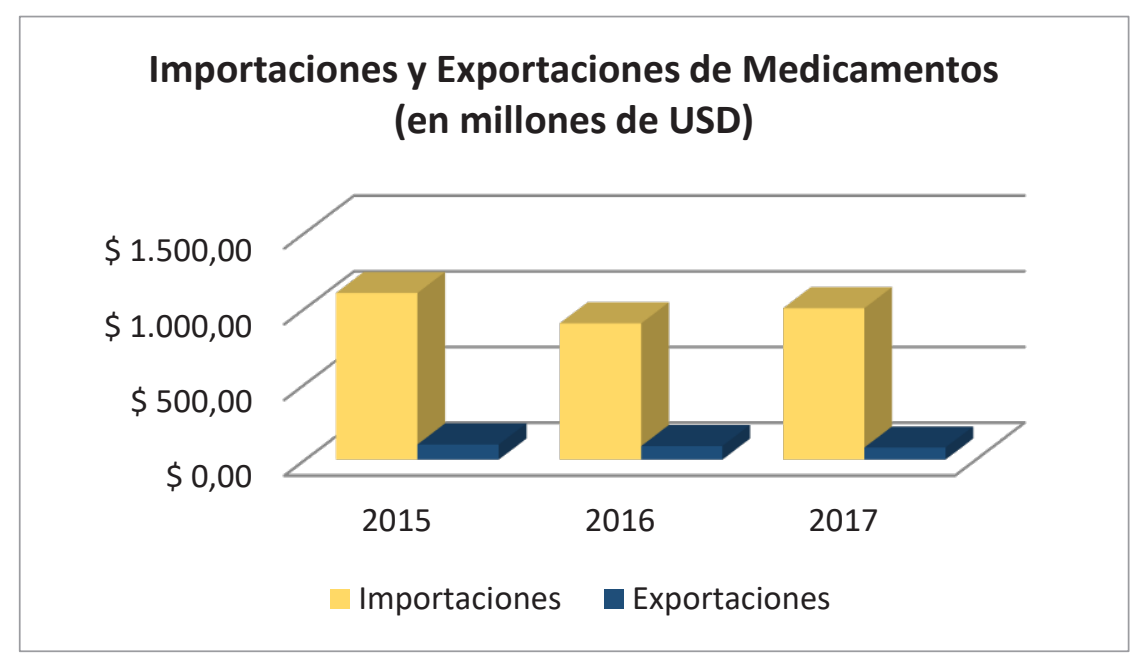

Fuente: Trade Map

Elaboración propia

Restricciones en la Importación de medicamentos

La partida arancelaria 3005.10.10.00 - - - Esparadrapos y venditas se encuentra actualmente restringida su importación a suelo ecuatoriano. 


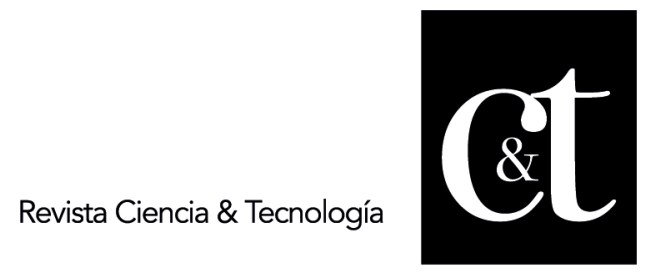

No. 33, 31 de enero de 2022

ISSN impreso: 1390 - 6321

ISSN online: 2661 - 6734

\section{Pasos Previos para Importar}

Obtención del Certificado digital para firma electrónica

El Banco Central del Ecuador ofrece es necesario para la firma electrónica de la declaración de importación

Instalación del Ecuapass

Registro de importador en sistema informático aduanero.

Conocer restricciones de productos a importar

Tramites de desaduanización de mercancías. (Servicio Nacional de Aduana del Ecuador , 2020)

\section{Industria Farmacéutica Local}

Para el caso de la industria farmacéutica local, las actividades de I\&D, Registro y Prescripción involucran la participación de actores ubicados fuera de la industria.

\section{I\&D}

En varios países del mundo, se realizan continuas actividades de investigación y desarrollo (I\&D) con la finalidad de la creación de un medicamento innovador que cure enfermedades que no tenían cura mediante la utilización de laboratorios de alta gama en los cuales disponen de los recursos necesarios para la investigación, donde cuentan con las capacidades científicas, tecnológicas y financieras, estas labores son de alto riesgo por ello la ejecución del mismo el cual requiere de un tiempo prolongado (10 a 15 años). (Comercio, 2013)

\section{Registro}

La Agencia Nacional de Regulación, Control y Vigilancia Sanitaria (ARCSA) es la institución que se encargara de asegurar la calidad, eficacia y seguridad de los fármacos. Además de ello, la agencia realiza controles de forma periódica posregistro sobre aquellos medicamentos que obtuviesen el certificado de registro sanitario. La vigencia del certificado de registro sanitario es de cinco años, luego de lo cual se debe iniciar el trámite de renovación automática por parte de ARCSA. (Comercio, 2013)

\section{Empresas que realizan actividades de distribución de fármacos.}

Respecto a la distribución de productos farmacéuticos, existen empresas muy reconocidas por su alta capacidad competitiva, entre ellos se destaca:

Grupos Holding (DIFARE y FYBECA)

Las ventas de estas grandes distribuidoras de medicamentos representan más de $55 \%$ de la totalidad del sector farmacéutico. Estas grandes cadenas de distribución "cuentan con una importante habilidad a la hora de negociar ante los laboratorios debido a la cantidad de compras que realizan (Antonio Quezada Pavón , 2011).

En la actualidad las grandes distribuidoras farmacéuticas administran alrededor del $64 \%$ del flujo de fármacos de los laboratorios, y controlan cerca del $89 \%$ de dicho flujo hacia las farmacias alrededor del país (Plaza, 2018).

\section{Marco legal}

Con base a las leyes ecuatorianas, y de acuerdo a los arts. 53 y 54 del Código Orgánico de la Producción, Comercio e Inversiones, en los cuales señala que la micro, pequeña y mediana empresa es toda aquella persona natural o jurídica que 


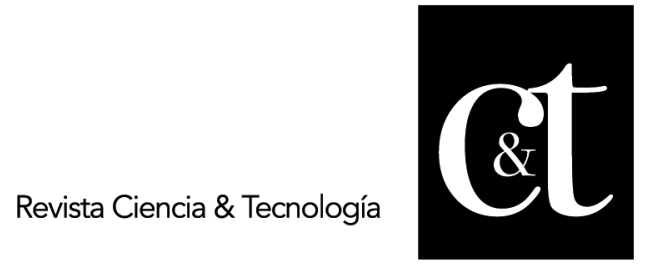

No. 33, 31 de enero de 2022

ISSN impreso: 1390 - 6321

ISSN online: 2661 - 6734

se considera como unidad productiva ejerciendo alguna actividad relacionada a la producción, comercio y servicios cumpliendo con las condiciones a considerarse como PYMES. (2018).

El marco legal que se considera para la industria del sector farmacéutico se concentra en el contenido de las siguientes disposiciones legales:

Constitución de la República.

Ley Orgánica del Sistema Nacional de Salud.

Ley de Producción, Importación, Comercialización y Expendio de Medicamentos Genéricos de Uso Humano.

Ley De Medicamentos Genéricos De Uso Humano

Ley Orgánica de Regulación y Control del Poder de Mercado. (Plaza, 2018)-

\section{Metodología}

Ante todo, se debe conocer el concepto de una investigación el cual es el procedimiento de indagación y curiosidad que se evidencia mediante la evidencia física que se plasma en un informe con la ayuda de la información recolectada por el cual se llega a obtener conocimiento científico, aunque no existe un método $100 \%$ infalible que pueda eliminar el margen de error que pueda darse en la elaboración del escrito. (Stenhouse, 2018)

En un sentido global, el autor Tomás Gallego Izquierdo (2007) los métodos son un conjunto de procedimientos que se llevan a cabo con la finalidad de encontrar la verdad y mostrarla, su principal objetivo es la solución de problemas mediante el uso y aplicación de técnicas e instrumentos. (pág. 84)

Existen muchos tipos de métodos de investigación que se pueden aplicar en un trabajo investigativo, sin embargo, solo se tomaran en cuenta aquellos métodos que son factibles en el presente trabajo. Se utilizará el método analítico-sintético para lograr identificar los factores limitantes que inciden en la negociación internacional de productos farmacéuticos de las PYMES, el cual de acuerdo al artículo el M.B.A Francisco Rodríguez (2007) se basa en el estudio de los hechos partiendo de la descomposición del objeto de estudio en cada una de sus partes para examinarlas de forma individual, analizando cada una para luego integrarlas todas juntas y estudiar su síntesis. (pág. 15)

Mediante la aplicación de la metodología de una investigación documental se recaudará una mayor cantidad de fuentes de información de alto reconocimiento e impacto académico relacionadas en el ámbito sectorial farmacéutico con el fin de evidenciar el aporte que éstos brindan a las PYMES.

\section{Unidad de análisis, población y muestra}

Para la presente investigación, se considera como objeto de estudio a la Importadora Jorua Cia. Ltda. Constituida en la ciudad de Babahoyo, provincia de Los Ríos y la población está definida por los empleados, jefes y gerente. En relación a la muestra, se considera el personal de la Importadora JORUA Cia. Ltda., seleccionando un total de 123 empleados, 7 jefes y 3 gerentes. 


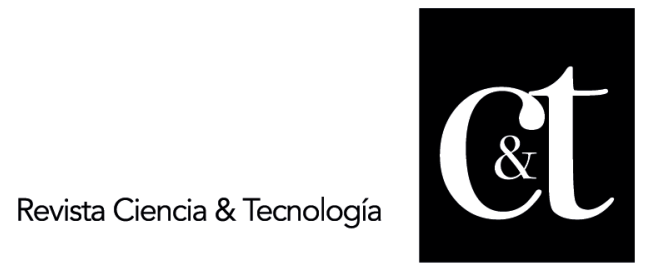

No. 33, 31 de enero de 2022

ISSN impreso: 1390 - 6321

ISSN online: 2661 - 6734

Se tomó en consideración como población total las 133 personas (empleados, jefes y gerentes) y se seleccionó una muestra de 46 personales tomando en cuenta principalmente a los gerentes, jefes y un selectivo grupo de empleados, la decisión tomada se basa en el muestreo intencional que es un tipo de muestreo no probalísticos el cual se toma en cuenta el juicio de valor del investigador debido a que esta persona será la encargada se escoger de forma personal a un cierto de personas capacitadas con la finalidad de que sea preciso el criterio de selección. (Sadornil, 2013)

Se tomó en consideración como población total las 133 personas (empleados, jefes y gerentes) y se seleccionó una muestra de 46 personales tomando en cuenta principalmente a los gerentes, jefes y un selectivo grupo de empleados, la decisión tomada se basa en el muestreo intencional que es un tipo de muestreo no probabilísticos el cual se toma en cuenta el juicio de valor del investigador debido a que esta persona será la encargada se escoger de forma personal a un cierto de personas capacitadas con la finalidad de que sea preciso el criterio de selección. (Sadornil, 2013).

\section{Variables de la investigación, operacionalización}

La presente investigación es de tipo no experimental, ya que, en esta, el investigador no tiene control sobre las variables independientes porque ya ocurrieron los hechos o porque son intrínsecamente manipulables; tiene que limitarse a la observación de situaciones ya existentes dada la incapacidad de influir sobre las variables y sus efectos.

Gemma García Ferre (2005) nos argumenta que "el propósito de una investigación exploratoria es proporcionar ideas que posteriormente se convertirán en un plan de acción investigativo, para ayudar a reconocer las variables relevantes. Es decir, este tipo de información es el inicio de un conjunto de ideales".

Por otra parte, para Mohammad Naghi Namakforoosh, (2005) y al contrario de la investigación exploratoria, la investigación descriptiva "es una forma de estudio para saber quién, cómo, cuando, donde y por qué del sujeto de investigación, la información obtenida se explica de forma correcta gracias al informe previo realizado" (pág. 91).

\section{Tabla 2 Variable independiente (VI) - Variable dependiente (VD)}

\begin{tabular}{|l|l|}
\hline $\begin{array}{l}\text { Variable independiente } \\
\text { (VI) }\end{array}$ & Variable dependiente (VD) \\
\hline Factores limitantes & Negociación internacional \\
\hline Causa & Efecto \\
\hline
\end{tabular}

Fuentes, técnicas e instrumentos para la recolección de información

El análisis de este proceso se enmarca en la metodología de una investigación documental, la búsqueda de la información se basa en los recursos de alto reconocimiento e impacto académico y científico, combinando campos de búsqueda con relacionados a las PYMES del sector farmacéutico.

José Alberto Yuni (2006), describe la técnica de la "observación" como "la inspección y estudio realizado por el investigador, mediante el empleo de sus propios sentidos, con 


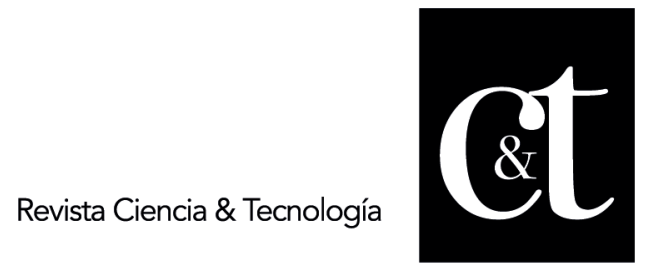

No. 33, 31 de enero de 2022

ISSN impreso: 1390 - 6321

ISSN online: 2661 - 6734

o sin ayuda de aparatos técnicos, de las cosas o hechos de interés social" (pág. 40). Las percepciones sobre los factores limitantes que inciden en la negociación internacional de productos farmacéuticos de las PYMES - caso Importadora Jorua Cia. Ltda., serán medidas a través de una encuesta, que es aplicable a través de un cuestionario establecido bajo la escala nominal, la cual comprende los datos nominales que no tienen un orden: los datos dicotómicos solo pueden tomar dos valores posibles (como vivo o muerto - sí o no). Con el propósito de examinar la validez de constructo de las escalas empleadas en el presente estudio, logrando realizar correcto análisis (S., 2014).

A través de la revisión en fuentes primarias, y secundarias se recabará información referente a los aportes que brindan las estrategias de internacionalización de los laboratorios farmacéuticos extranjeros a las PYMES - caso Importadora Jorua Cia. Ltda., para ello se utilizará la técnica de investigación documental.

Entre las fuentes utilizadas, están:

\section{Fuentes primarias}

En este grupo se identifican los datos que provienen de:

Informes del Banco Central del Ecuador.

Informes del Ministerio de Salud Pública.

Bases de Datos de la Agencia Nacional de Regulación, Control y Vigilancia Sanitaria ARCSA.

Informes de la Organización Mundial de Comercio.

Informes de la Superintendencia de Compañías, Valores y Seguros.

Bases de datos del Instituto Nacional de Estadísticas y Censos - INEC.

\section{Fuentes secundarias}

También se requirió información externa que aporta con datos importantes para la investigación, identificando las siguientes fuentes secundarias:

Artículos científicos a nivel local, regional y mundial de revistas científicas.

Trabajos investigativos publicados en tesis de grado, posgrado y doctorado.

Libros y secciones de libros de gestión empresarial, Pymes, relacionados a la industria farmacéutica.

\section{Tratamiento de la información}

Para el análisis de la información de forma estadística, se realizará el análisis descriptivo y pruebas estadísticas que permitan determinar los factores limitantes que inciden en la negociación internacional de productos farmacéuticos de las PYMES - caso Importadora Jorua Cia. Ltda.

Con el propósito de examinar la validez de la información es aplicable un cuestionario establecido bajo la escala nominal, la cual comprende los datos nominales que no tienen un orden: los datos dicotómicos solo pueden tomar dos valores posibles (como vivo o muerto - sí o no) constructo de las escalas empleadas en el presente estudio se realizará un análisis factorial. A través de la revisión en fuentes primarias, se recabará información referente a las PYMES farmacéuticas de la ciudad de Babahoyo, para lo cual se utilizarán datos de la Superintendencia de Compañía, Servicio de Rentas Internas y Cámara de Comercio. 


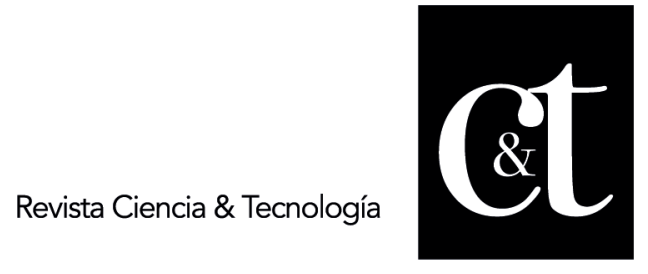

No. 33, 31 de enero de 2022

ISSN impreso: 1390 - 6321

ISSN online: 2661 - 6734

Con el fin de determinar la liquidez de la Importadora Jorua Cia. Ltda. Se efectuará un análisis financiero, partiendo de los estados financieros y estados de resultados de los tres últimos años, con el propósito de evidenciar la evolución de la situación financiera de las mismas.

\section{Análisis de la situación actual}

El estudio de la situación sobre los limitantes de la negociación internacional expresa que los problemas que se pueden presentar son a considerarse, en especial para las empresas que dependen de cierto tipo de comercio y obtienen, ya sea materia prima o productos terminados en el extranjero.

La empresa JORUA CIA LTDA al ser una de las Pymes en el Ecuador que efectúa la actividad de importación de productos farmacéuticos funge como objeto de estudio para la comprobación de las interrogantes diseñadas para detectar si las restricciones internacionales afectan a la adquisición de los productos, para lo cual se ha determinado la realización de 2 métodos para la determinación de los resultados.

Encuesta ejecutada a los Trabajadores de la empresa.

Análisis Financiero a la empresa Jorua Cia Ltda.

Encuesta

Para la identificación de la situación actual de la empresa de estudio JORUA CIA. LTDA, se presentan los resultados obtenidos mediante la aplicación de la encuesta ejecutada a los trabajadores de la empresa relacionados al área contable, administrativa o gerencial.

La presentación de los resultados se realiza de forma ordenada con la finalidad de describir y mostrar de manera sintética el análisis obtenido de cada respuesta expresado por los funcionarios de la empresa.

La encuesta consiste en un formato de 8 preguntas planificadas y diseñadas con el fin de obtener información que ayude a solucionar las interrogantes planteadas. La población encuestada consiste en los trabajadores de la empresa como se mencionó antes, los mismos se encuentra ciertamente vinculados con el proceso de importación de productos farmacéuticos.

\section{Presentación de resultados y discusión}

Las encuestas que han sido aplicadas al personal de la empresa tanto administrativo como contable reflejan los siguientes resultados:

Los limitantes para la negociación internacional de productos farmacéuticos en la empresa, si representa una dificultad a la hora de adquirir el producto, por lo que suelen existir retrasos ocasionales en este proceso cada vez que se va a realizar una nueva adquisición.

Al ser la adquisición o importación de productos fármacos un proceso con complicación, se ha optado en la empresa por cambio la distribución de los productos y usar productos nacionales.

La importación de las mercaderías (fármacos) es un proceso conocido por la mayoría de la empresa, dado que de este se depende el curso que este tome, ya que la venta del mismo es una de las que más genera recursos en general en Jorua Cia. Ltda.

De acuerdo a la expresión de los trabajadores de la empresa, el resultado económico de la misma ha sido afectado, ya que muchas veces no se puede realizar la adquisición de productos fármacos por complicaciones vinculadas a las limitaciones antes mencionadas. 


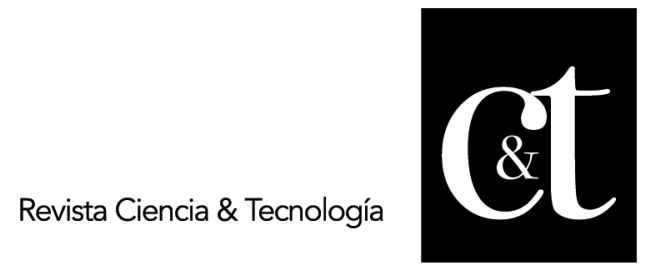

No. 33, 31 de enero de 2022

ISSN impreso: 1390 - 6321

ISSN online: 2661 - 6734

La aparición de la pandemia causada por el coronavirus Covid-19 se ha manifestado como una nueva limitación para la importación de productos farmacéuticos hacia la empresa, como se lo pudo reflejar en las conceptualizaciones tomadas, donde se asegura que muchos países evitan la exportación de sus medicinas para salvaguardar las vidas de sus habitantes.

Muchos de los requisitos que se solicitan por parte del Estado y Aduana, es visto como una complicación, ya que muchos de estos requisitos son difíciles de conseguir, aun para la empresa que ya está constituida, por lo que el proceso se muestra aún más dificultoso para una Pyme que apenas inicie en este negocio.

Por otro lado, la ejecución del estudio financiero a los Estados de la Empresa arrojó los siguientes resultados:

Los resultados financieros de la empresa demuestran que la empresa no posee la liquidez suficiente para afrontar las deudas durante los periodos 2018 y 2019 , problema que puede estar vinculado a la problemática planteada como uno de los factores que influyen en la liquidez de la empresa.

Existen cambios significativos en la empresa, a nivel económico, o lo que sugiere que esta ha estado creciendo en inversión, sin embargo, no se ha obtenido el nivel de liquidez esperado.

Durante el periodo 2019 al 2020, la empresa al realizar una inversión en el stock de la mercadería ha conseguido una mejoría significativa, apoyándose en obtener una mejor distribución del producto que pueda abarcar más lugares, sin embargo, la aparición de nuevas restricciones o limitaciones sobre la adquisición de mercadería, podría generar que la inversión resulte en contra de la empresa, en caso de que las medidas se lleguen a tornar muy dificultosas.

\section{Conclusiones}

El estudio de la situación sobre los limitantes de la negociación internacional expresa que los problemas que se pueden presentar son a considerarse, en especial para las empresas que dependen de cierto tipo de comercio y obtienen, ya sea materia prima o productos terminados en el extranjero.

La empresa JORUA CIA LTDA al ser una de las Pymes en el Ecuador que efectúa la actividad de importación de productos farmacéuticos funge como objeto de estudio para la comprobación de las interrogantes diseñadas para detectar si las restricciones internacionales afectan a la adquisición de los productos, para lo cual se ha determinado la realización de 2 métodos para la determinación de los resultados.

Las encuestas que han sido aplicadas al personal de la empresa tanto administrativo como contable reflejan resultados que son limitantes para la negociación internacional de productos farmacéuticos en la empresa, si representa una dificultad a la hora de adquirir el producto, por lo que suelen existir retrasos ocasionales en este proceso cada vez que se va a realizar una nueva adquisición. Al ser la adquisición o importación de productos fármacos un proceso con complicación, se ha optado en la empresa por cambio la distribución de los productos y usar productos nacionales. 


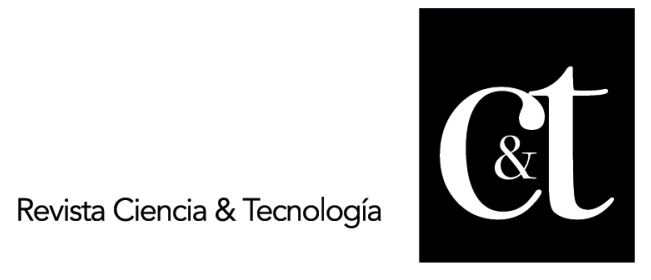

No. 33, 31 de enero de 2022

ISSN impreso: 1390 - 6321

ISSN online: 2661 - 6734

La importación de las mercaderías (fármacos) es un proceso conocido por la mayoría de la empresa, dado que de este se depende el curso que este tome, ya que la venta del mismo es una de las que más genera recursos en general en Jorua Cía. Ltda. De acuerdo a la expresión de los trabajadores de la empresa, el resultado económico de la misma ha sido afectado, ya que muchas veces no se puede realizar la adquisición de productos fármacos por complicaciones vinculadas a las limitaciones antes mencionadas.

La aparición de la pandemia causada por el coronavirus Covid-19 se ha manifestado como una nueva limitación para la importación de productos farmacéuticos hacia la empresa, como se lo pudo reflejar en las conceptualizaciones tomadas, donde se asegura que muchos países evitan la exportación de sus medicinas para salvaguardar las vidas de sus habitantes. Muchos de los requisitos que se solicitan por parte del Estado y Aduana, es visto como una complicación, ya que muchos de estos requisitos son difíciles de conseguir, aun para la empresa que ya está constituida, por lo que el proceso se muestra aún más dificultoso para una Pyme que apenas inicie en este negocio.

Por otro lado, la ejecución del estudio financiero a los Estados de la Empresa arrojó los resultados financieros de la empresa demuestran que la empresa no posee la liquidez suficiente para afrontar las deudas durante los periodos 2018 y 2019, problema que puede estar vinculado a la problemática planteada como uno de los factores que influyen en la liquidez de la empresa. Existen cambios significativos en la empresa, a nivel económico, o lo que sugiere que esta ha estado creciendo en inversión, sin embargo, no se ha obtenido el nivel de liquidez esperado. Durante el periodo 2019 al 2020, la empresa al realizar una inversión en el stock de la mercadería ha conseguido una mejoría significativa, apoyándose en obtener una mejor distribución del producto que pueda abarcar más lugares, sin embargo, la aparición de nuevas restricciones o limitaciones sobre la adquisición de mercadería, podría generar que la inversión resulte en contra de la empresa, en caso de que las medidas se lleguen a tornar muy dificultosas

\section{Referencias bibliográficas}

(CEPAL), C. E. (2020). Las restricciones a la exportación de productos médicos dificultan los esfuerzos por contener la enfermedad por coronavirus (COVID-19) en América Latina y el Caribe. Chile.

Quezada Pavón Antonio . (enero de 2011). La Industria Farmacéutica en el Ecuador: Mirando Hacia Adelante. Obtenido de http://www.espae.espol.edu.ec/: https://www.espae.espol.edu.ec/wpcontent/uploads/2011/12/EyE_Industria_Farmaceutica_2011.pdf

Bank watch ratings S.A . (Enero de 2019). Bank watch ratings S.A - Calificadora de Riesgo. Obtenido de Bank watch ratings S.A - Calificadora de Riesgos : https://www.bankwatchratings.com/noticias/187-sectorial-farmaceutico-enero2019

CODIGO ORGANICO DE LA PRODUCCION, COMERCIO E INVERSIONES, COPCI. (21 de agosto de 2018). Correos del Ecuador. Obtenido de https://www.correosdelecuador.gob.ec/:

https://www.correosdelecuador.gob.ec/wpcontent/uploads/downloads/2018/11/COPCI.pdf 


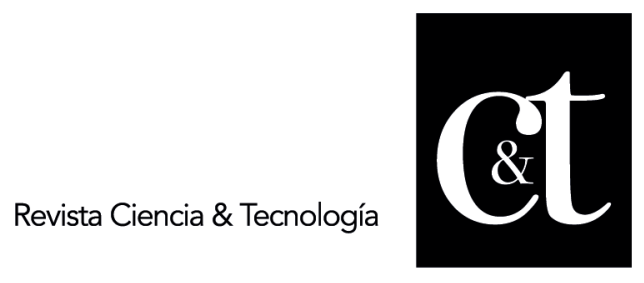

No. 33, 31 de enero de 2022

ISSN impreso: 1390 - 6321

ISSN online: 2661 - 6734

Comercio, E. (20 de Agosto de 2013). El Comercio. Obtenido de https://www.elcomercio.com/: https://www.elcomercio.com/tendencias/ricardoaman-50-poblacion-urbana.html

Rodríguez C. Francisco, M.B.A. (2007). GENERALIDADES ACERCA DE LAS TÉCNICAS DE INVESTIGACIÓN CUANTITATIVA. Paradigmas, Vol 2, No. 1 , pp. 9 - 39.

González, P. C. (8 de Agosto de 2017). Diario El Comercio. Obtenido de EL COMERCIO: https://www.elcomercio.com/actualidad/importaciones-sector-farmaceuticosupera-exportaciones.html

Plaza, M. A. (6 de Abril de 2018). Espae.Espol. Obtenido de http://www.espae.espol.edu.ec/: $\quad$ http://www.espae.espol.edu.ec/wpcontent/uploads/documentos/IndustriaFarmaceutica.pdf

Sadornil, D. D. (2013). DICCIONARIO-GLOSARIO DE METODOLOGÍA DE LA INVESTIGACIÓN SOCIAL. Madrid: uned.

Servicio Nacional de Aduana del Ecuador. (Febrero de 2020). https://www.aduana.gob.ec/. Obtenido de https://www.aduana.gob.ec/paraimportar/

Stenhouse, L. (2018). La investigación como base de la enseñanza. Madrid: Morata.

Gallego Izquierdo Tomás. (2007). Bases teóricas y fundamentos de la fisioterapia. Madrid: editorial medica panamericana.

Valencia Olvera, K. L. (2017). Control de Inventarios de Producción en la Importadora Jorua Cia tda y su Rentabilidad. Babahoyo, Los Rios, Ecuador: Universidad Tecnica de Babahoyo. 